\title{
EQUIPE DE ENFERMAGEM: ANÁLISE DO CLIMA ORGANIZACIONAL NO CENTRO CIRÚRGICO DO HOSPITAL UNIVERSITÁRIO GETÚLIO VARGAS
}

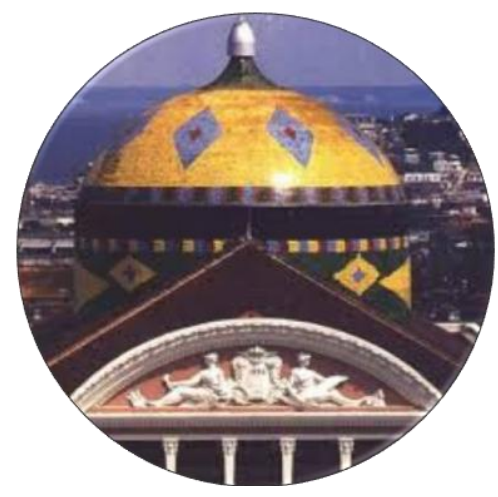

${ }^{1}$ Jaqueline Alves Chaves
${ }^{2}$ Maria da Gloria Vitório Guimarães

\section{Resumo}

A qualidade do ambiente organizacional influência cada vez mais o comportamento dos colaboradores, interferindo na performance e nos resultados, por conseguinte, objetiva-se conhecer os fatores favoráveis a um bom clima organizacional no âmbito da equipe de enfermagem do centro cirúrgico de um hospital. Buscou-se examinar a opinião dos enfermeiros, técnicos e auxiliares de enfermagem num total de 46, a respeito das dez dimensões consideradas relevantes a um ambiente saudável. Para tanto foi aplicado um questionário contendo 60 questões. As análises foram quantitativas, desenvolvidas a partir do software SPSS. Os resultados demonstraram que os servidores não estão satisfeitos com a segurança e as condições do trabalho, indicaram que os treinamentos sãoescassos, além disso, não existe reconhecimento quando o trabalho é bem feito.

Palavras-chave: Clima organizacional.Comportamento humano. Equipe de enfermagem. Hospital público.

\begin{abstract}
The quality of the organizational environment increasingly influences the behavior of employees, interfering in performance and results, therefore, it is aimed to know the

${ }^{1}$ Mestre em Engenharia de Produção, Servidora Técnico Administrativo da Universidade Federal do Amazonas.

2 Professora Adjunta da Universidade Federal do Amazonas, Doutora em Ciências/Psicologia pela Universidade de São Paulo, Tutora do Programa de Educação Tutorial do MEC, Avaliadora do INEP-MEC, Oito anos coordenando cursos de Pós-Graduação Lato Sensu/UFAM.
\end{abstract}


factors favorable to a good organizational climate within the nursing team of the surgical center of a hospital. It was sought to examine the opinion of nurses, technicians and nursing assistants out of a total of 46, regarding the ten dimensions considered relevant to a healthy environment. A questionnaire containing 60 questions was applied. The analyzes were quantitative, developed from SPSS software. The results showed that the servers are not satisfied with the safety and working conditions, indicated that the training is scarce, and there is no recognition when the work is done well.

Keywords: Organizational climate. Human behavior. Nursing team. Public hospital.

\section{INTRODUÇÃO}

Observa-se que nos serviços da maioria das instituições de saúde, está presente a assistência de enfermagem ao paciente, uma vez que, a enfermagem trabalha com os pacientes desde os primeiros sintomas da doença. O oferecimento de serviços de saúde implica em contato e interação entre o profissional, o paciente e a instituição, sendo estes fatores fundamentais para a prática de uma boa assistência, pois ambos participam mutuamente no estabelecimento do quadro clínico do paciente. "O trabalho hospitalar envolve, essencialmente, o trabalho de equipe" (BACKES; LUNARDIFILHO, 2006, p.1060), por conseguinte, uma estrutura física adequada, equipamentos de proteção, treinamentos constantes e boas relações de trabalho são bastante importantes.

Para Srour (1998, p. 86), o clima é uma das variáveis que influência o desempenho organizacional no processo de alcance dos objetivos propostos. Nas unidades de saúde é primordial um ambiente propício à integração das equipes multifuncionais, bem como promoção da qualidade de vida no trabalho, motivação, satisfação e possibilidades de desenvolvimento. Portanto, torna-se prioritário uma análise do clima organizacional que possa identificar a percepção e opinião dos servidores sobre o clima existente. A pesquisa de clima organizacional deve ser considerada como uma estratégia para identificar oportunidades de melhorias contínuas no ambiente de trabalho e na produtividade. 
Entende-se que a administração do clima é uma responsabilidade da administração de gestão de pessoas, pois as inadequações ao trabalho, desajustes comportamentais e estresse são aspectos que interferem na vitalidade, ação e proação das pessoas. No Hospital Universitário Getúlio Vargas (HUGV) cada clínica é gerenciada por uma enfermeira denomina de chefa da equipe de enfermagem, com formação em enfermagem, todavia sem preparo para gestão de pessoas, desse modo, objetiva-se avaliar e conhecer o Clima Organizacional no âmbito do ambiente da equipe de enfermagem do centro cirúrgico do Hospital Universitário Getúlio Vargas, a fim de apresentar sugestões que possam melhorar o desempenho da equipe e a assistência ao paciente.

\section{CLIMA ORGANIZACIONAL}

Administrar o clima no âmbito organizacional é uma ação estratégica que deve ser observada por todos os membros da organização, contudo muitas ações são intervenções superficiais, voltadas para resolver problemas imediatos, como resultado, não proporcionam condições de desenvolvimento e equilíbrio psicológico. Acrescenta-se a isso, as limitações de análise organizacional para diagnosticar situações que exigem uma compreensão mais abrangente da natureza do trabalho humano (STONER; FREEMAN, 1999, p. 17).

Desse modo, "o clima representa o ambiente psicológico e social que existe em uma organização e que condiciona o comportamento dos seus membros" (CHIAVENATO, 2005, p. 123). "O clima pode se referir ao ambiente dentro de um departamento, de uma fábrica ou de uma empresa" (CHIAVENATO, 2005, p. 124).

Logo, a origem da palavra organização que deriva do grego

organon, significa uma ferramenta ou instrumento, "nesse sentido as organizações podem ser entendidas como instrumentos utilizados pelo 
homem para desenvolver determinadas tarefas, não possíveis para um indivíduo em particular" (DIAS, 2003, p.10). Na perspectiva mais conhecida e aceita se entende organização "como um ente social criado intencionalmente para se conseguir determinados objetivos mediante o trabalho humano e uso de recursos materiais" (DIAS, 2003, p. 22).

Para Drucker, “organização é um grupo humano, composto por especialistas que trabalham em conjunto em uma tarefa comum" (1999, p. 61). Em virtude disso, o elemento humano separado dos grupos e das organizações não constitui uma célula social. Assim, também, as instituições sem o elemento humano não constitui uma empresa. A empresa é o conjunto do homem e do patrimônio, estas afirmativas são verdades que têm evidência, por si mesmas, não necessitando de demonstração, são axiomáticas (SÁ; LEMOINE, 1999, p. 45).

Portanto, a estrutura organizacional juntamente com a cultura existente e a organização do trabalho estabelecida resultará no clima organizacional da instituição. A cultura organizacional representa as normas informais e não escritas que orientam o comportamento dos membros da organização no dia a dia e que direcionam suas ações para a realização dos objetivos organizacionais (CHIAVENATO, 2005, p. 35).

No que diz respeito, ao Clima Organizacional entende-se como a expressão dos sentimentos dos colaboradores diante dos valores, das políticas, das práticas de gestão das pessoas, da forma de relacionamento com os colegas, da postura da empresa ao estabelecer e retribuir pelo alcance das metas, além da situação econômica (BOOG, 2002, p. 10).

Conforme a concepção de Dias (2003, p. 67) pode-se dizer que na composição do Clima Organizacional, é importante observar:

- Clima de intimidade: favorece a integridade do grupo e favorece relações sociais amistosas. Estas relações devem satisfazer as necessidades sociais de relacionamento e não estão necessariamente ligadas a tarefa desenvolvida no local de trabalho; 
- Clima de espírito de equipe: os membros da organização sentem que suas necessidades sociais são atendidas e, ao mesmo tempo, gozam do sentimento de tarefa cumprida;

- Clima com ênfase na produção: os integrantes sentem que seu comportamento no trabalho é bastante supervisionado, ademais recebe pressão por resultados. A administração é fortemente direcionada ao seu objetivo principal (metas e produção) e muitas vezes insensível aos problemas e opiniões individuais;

- Clima burocrático: há uma atmosfera fechada e formal. Insiste-se na papelada e canais regulamentares. Nas discussões de trabalho, predominam referências às regras, regulamentos e procedimentos;

- Clima de cordialidade: ocorre um sentimento geral de camaradagem que prevalece na atmosfera do grupo de trabalho, havendo maior ênfase nas pessoas e uma predominância de grupos sociais amistosos e cordiais;

- Clima de tolerância: deriva para um comportamento organizacional onde os erros são tratados como forma de apoio e aprendizagem, todavia pode existir uma atmosfera ameaçadora, punitiva ou inclinada a colocar a culpa em alguém.

Partindo deste contexto, foram analisadas determinadas dimensões sobre clima organizacional, sempre com foco em uma unidade hospitalar pública.

\section{DIMENSÕES DE CLIMA ORGANIZACIONAL}

\subsection{Cooperação e adesão}

"O homem enfrenta continuamente dificuldades na convivência com o outro, o que gera conflitos nas situações práticas do cotidiano" 
(KANAANE, 2012, p. 113). As pessoas se apoiam mutuamente quando há concordância autêntica e discordam entre si em caso contrário. A cooperação pode ser funcional ou disfuncional para o sistema organizacional, se as reflexões e as concordâncias são autênticos esforços de integração e aprendizagem, tem-se um processo funcional, ao contrario disso, se as pessoas encobrem discordâncias que precisam ser expostas para se chegar a decisões equilibradas, a cooperação é disfuncional (COHEN, 2003, p. 28). Desse modo, pode-se observar que quanto maior for o grau de interação entre os grupos ou entre as pessoas de um dado setor de trabalho, maior será a tendência à cooperação mútua, simultaneamente, tem-se um clima propicio ao aumento da eficácia.

\subsection{Segurança no trabalho}

Segurança no trabalho é o conjunto de medidas que visam a prevenir acidentes. Constitui, pois, um dos fatores decisivos para o aumento da produtividade, na medida em que reduz o número de faltas decorrentes de condições de trabalho inadequadas (TACHIZAWA; FERREIRA; FORTUNA, 2001, p. 102).

Ainda segundo os autores, o primeiro passo para prevenir os acidentes de trabalho é identificar as suas causas. As principais causas de acidentes são: características pessoais inadequadas; comportamentos disfuncionais, como desatenção, esquecimento, negligência e imprudência; degradação do ambiente de trabalho, devido a fatores potencialmente causadores de acidentes, como equipamentos mal projetados ou em precário estado de conservação e arranjo físico mal definido. 


\subsection{Condições no trabalho}

Conforme Kanaane (2012, p. 34), o trabalho é uma ação humana exercida num contexto social, que sofre influências oriundas de distintas fontes, o que resulta numa ação recíproca entre o colaborador e os meios de trabalho. As condições no trabalho deve possibilitar o surgimento de possibilidades facilitadoras para a adaptação e ajustamento do individuo, quer como pessoa, quer como profissional, com um incremento efetivo à produtividade. Isto implica, necessariamente, em conhecer e redefinir o sistema de trabalho através da flexibilização das relações de poder, autoridade e comunicação.

\subsection{Habilidade de supervisão}

As unidades hospitalares para serem efetivas, precisam de supervisores ágeis, adaptáveis, flexíveis as necessidades, que detenham as novas técnicas de liderança. Há uma expectativa de que os enfermeiros de todas as clínicas deleguem e supervisionem o trabalho de diferentes profissionais da saúde: auxiliares e técnicos de enfermagem, técnicos de nutrição, lavanderia, manutenção e os serviços prestados durante a residência médica. Para que esses profissionais desempenhem o papel de supervisores e gerentes precisam de preparo para assumir tais tarefas de liderança (MARQUIS; HUSTON, 2005, p. 30).

\subsection{Gestão de pessoas}

A gestão de pessoas deve se preocupar em treinar, avaliar, reter talentos e gerenciar os recursos humanos de forma eficiente e eficaz, buscando os melhores resultados. 
Poucas instituições contam com a variedade de profissões, encargos, diversidade de tarefas, pressões e riscos pessoais de um hospital. Também são poucas as instituições nas quais o trabalho é permanente e intenso, durante as vinte e quatro horas do dia, todos os dias do ano, com atividades inesperadas a cada dia e cujas situações de emergência surgem com frequência e comportam risco (LONDOÑO; MORERA; LAVERDE, 2003, p. 32). Desse modo, o hospital apresenta características complexas e de difícil administração em termos de recursos humanos. O universo de profissionais existentes para a execução das atividades dentro de um hospital é muito amplo, são realizados trabalhos, médico-cirúrgico, assistência de enfermagem, serviços de laboratório, exames, lavanderia, alimentação, almoxarifado, informática, e outros. Enfim, o quadro de pessoal inclui o corpo assistencial, os funcionários da área administrativa e de suporte operacional (ZANON, 2001, p. 94). Os quais apresentam carências de preparo profissional, necessitando de acompanhamento e treinamento constante.

\subsection{Ambiente de trabalho}

As instituições bem sucedidas incentivam cada vez mais as discussões em grupo, o trabalho em equipe e a cooperação, buscando impulsionar um bom clima organizacional e reduzir ruídos de comunicação. As instituições e os profissionais são responsáveis pelo ambiente de trabalho, todavia, estresse, mau humor, chefias mal preparadas são ocorrências recorrentes no mundo moderno em razão da hiperatividade e do individualismo exacerbado. Estes processos além de prejudicarem a performance profissional, eles refletem diretamente na vida pessoal, portanto, o profissional poderá contribuir positiva ou negativamente nesse processo de trabalho. Para se ter um ambiente de trabalho equilibrado, é fundamental que o espaço hospitalar seja estimulante, descontraído e justo, 
onde exista respeito e os colaboradores possam se dedicar as atividades, sem prejudicar a sua saúde física e mental.

\subsection{Valorização e reconhecimento}

Em todo hospital, há uma variedade de chefes, muitos autocráticos e outros democráticos, bem como, descompassos entre o que deveria acontecer e o realmente acontece. As enfermarias possuem chefes distintos, acarretando conflitos e pressões constantes. Superiores autoritários geram desequilíbrio, medo e erros ao passo que os democráticos buscam descobrir o que há de melhor em cada funcionário, facilitam o alcance das metas, além disso, utilizam sua influência para melhorar a motivação que beneficia trabalhadores e, pacientes.

Chiavenato (2005, p. 202) alerta, toda pessoa precisa receber retroação, seja positiva ou negativa a respeito de seu desempenho. Sem essa retroação, as pessoas caminham às cegas, por conseguinte, os gestores devem aprender a reconhecer e respeitar os esforços individuais e recompensar adequadamente, desta forma pode-se alcançar níveis elevados de produtividade.

\subsection{Gestão}

A administração de um hospital envolve uma complexa associação de recursos humanos, materiais e serviços diversificados. Tais como os serviços de ambulância; manutenção hidráulica, elétrica e predial; serviços de alimentação e lavanderia que atuam conjuntamente com a complexidade dos cuidados médicos - cirúrgicos e de enfermagem.

Os hospitais públicos são organizações tradicionais, que apresentam forte resistência a mudanças, porque estas quase sempre implicam em alterações na distribuição de poder na instituição (ZANON, 
2001, p. 88). O sucesso de um bom chefe pode ser medido pelo desempenho e pelo resultado do setor, ademais, a soma da produtividade de cada pessoa mede a do seu chefe.

Segundo Motta e Vasconcelos (2008, p. 34), a gestão de pessoas tem, assim, o seguinte papel: avaliar as necessidades e os recursos dos grupos organizacionais; descrever os cargos e provê-los segundo um recenseamento sistemático dos candidatos, por meio de procedimentos de seleção; avaliar os cargos e desempenho dos empregados, remunerando-os equitativamente para motivá-los e treinar os indivíduos, melhorar as condições de trabalho, informar, comunicar e assegurar relações sociais satisfatórias.

\subsection{Comunicação}

Comunicação é o ato de comunicar, de transmitir, de participar (ZANON, 2001, p. 83). Consiste na troca de mensagens entre indivíduos através de símbolos e sinais que constituem a linguagem falada ou escrita. Para todo profissional de saúde, a comunicação com seus semelhantes é uma necessidade imperiosa (LONDOÑO; MORERA; LAVERDE, 2003, p. 75). Dessa forma, a comunicação facilita a motivação por esclarecer aos funcionários o que deve ser feito, qual a qualidade do seu desempenho e o que fazer para melhorá-lo (ROBBINS, 2005, p. 103). É importante analisar que a comunicação em qualquer instituição está sempre sujeita a distorções e mau-entendimentos muito embora, é crucial a comunicação entre as pessoas.

Há um clima interno e um externo na comunicação. O clima interno inclui valores, sentimentos, temperamento e níveis de estresse do emissor e do receptor. Os desentendimentos podem bloquear o desenvolvimento de uma relação e criar tensões em um relacionamento. Já as condições atmosféricas, temperatura, momento certo e ambiente da 
organização são partes do clima externo (MARQUIS; HUSTON, 2005, p. 87).

\subsection{Parceria}

A organização pode manter um clima de distanciamento e negativo, como pode criar solidariedade, calor humano, boa camaradagem e apoio à iniciativa pessoal e grupal. Quanto mais harmoniosa, tanto melhor o clima e as parcerias internas. "As boas ou más relações humanas no ambiente hospitalar são facilmente percebidas e decodificadas pelos pacientes, familiares e comunidade em geral” (BACKES; LUNARDIFILHO2006, p.1061). Sendo assim, é necessário que exista um bom relacionamento entre os membros da equipe de enfermagem, proporcionando assim crescimento, bem estar e aumento das contribuições, resultando em parceria no decorrer das atividades realizadas.

Dejours, Abdouchele e Jayet (2007, p. 69) advertem: se um trabalho permite a diminuição da carga psíquica, ele é equilibrante, por outro lado se ele se opõe a essa diminuição, ele é fatigante. Paralelamente o clima organizacional que não possibilita parceria e solidariedade a energia psíquica se acumula, tornando-se fonte de tensão e desprazeres

Desse modo, em um ambiente do qual várias pessoas participam diariamente para promover assistência e recuperação da saúde, faz-se necessário parceria. Segundo Baggio (2007, p. 78), no dia a dia da equipe de enfermagem, observa-se um clima de insatisfação, frequentes desentendimentos e ressentimentos entre os profissionais, ficando para trás o possível trabalho harmonioso em parceria. 


\section{INSTITUIÇÃO}

Os hospitais encontram-se entre as organizações mais complexas que existem, funcionam através da conexão e sinergia de cada uma das áreas envolvidas e da necessidade de harmonizar os processos ao plano final almejado, como resultado, deve traduzir em ações diárias o discurso do bem-estar e da saúde (MARX, 2003, p. 55). Uma vez que as organizações prestadoras de serviços, as instituições de saúde representam sistemas psicossociais, bem diferentes das organizações encarregadas da produção ou da comercialização de bens (GONÇALVES, 1987, p. 26).

Portanto, os hospitais apresentam-se como ambientes desafiadores para manutenção de um bom clima organizacional devido a multiplicidade da equipe de profissionais existentes nestes ambientes, faz-se necessário que esses profissionais sejam adequadamente 'cuidados' pelas instituições. Importa às instituições de saúde, determinar estratégias que vislumbrem a satisfação da equipe de colaboradores, ou seja, seu cliente interno.

$\mathrm{Na}$ cidade de Manaus existe o Hospital Universitário Getúlio Vargas (HUGV), que é público federal, do tipo hospital-escola, serve como campo de estágio para alunos da Escola de Enfermagem, Faculdade de Medicina, Odontologia e Farmácia da Universidade Federal do Amazonas. Galvão (2003, p.212) aponta:

A construção do Hospital Universitário Getúlio Vargas começou em 1962, durante o primeiro mandato do governo de Gilberto Mestrinho, e naquele momento, já se cogitava a possibilidade do HUGV vir a ser um hospital-escola dedicado ao ensino e formação de profissionais da área médica, fato que veio a ocorrer somente na década de 70 .

A inauguração do HUGV aconteceu no dia 27 de junho de 1965, no governo do professor Arthur Cezar Ferreira Reis, cujo secretário de 
Saúde, era o doutor Theomário Pinto da Costa (GALVÃO, 2003, p.14). Em 1966, a instituição teve suas instalações disponibilizadas para estágios e residências dos alunos do curso de medicina (BRITO, 2009, p. 37). Nessa época o HUGV foi considerado um dos maiores e melhores hospitais do Nortedo país, pois os equipamentos foram escolhidos através de uma equipe formada pelos mais importantes profissionais da área de saúde do Amazonas, porém ainda não existia mão-de-obra qualificada para manusear tais equipamentos, resultando em furtos, subutilização e devoluções (GALVÃO, 2003, p. 21).

No dia 4 de fevereiro de 1983, através do Decreto $n^{\circ}$ 6.994, o Governador Paulo Nery doou à Fundação Universidade do Amazonas o hospital e seu patrimônio, conforme escritura pública passada no Cartório de Registro de Imóveis $-2^{\circ}$ Oficio assinada pelo Procurador Geral do Estado, Osmar Pedrosa e o Professor Octávio Hamilton Botelho Mourão, Presidente da Fundação Universidade do Amazonas (BRITO, 2009, p.16).

A finalidade do hospital universitário de Manaus concentrava-se na prestação de assistência à saúde para população do Estado do Amazonas em seu nível de competência e, simultaneamente, oferecer campo de estágio para a formação de profissionais ao nível de graduação e de pósgraduação na área de saúde (REGIMENTO, 1995, p. 56).

Ao longo dos anos ocorreram mudanças na estrutura física da instituição, tais como: criação do centro cirúrgico, do ambulatório Araújo Lima e do núcleo de nutrição, implantação da farmácia hospitalar e a restauração do pronto-socorro (GALVÃO, 2003, p. 48), buscando tornar o hospital mais ágil e atualizado para responder aos novos desafios.

Atualmente a instituição está subordinada administrativamente à reitoria da Universidade Federal do Amazonas, exercendo atividades como hospital geral de atenção terciária denominada em alta complexidade (LOPES, 2006, p. 10). Logo, o HUGV executa programas de residências médicas em 15 especialidades: neurologia, ginecologia e obstetrícia, 
pediatria, cirurgia geral, anestesiologia, dermatologia, oftalmologia, nefrologia, anatomia patológica, clinica médica, doenças infecciosas parasitarias, ortopedia, urologia, medicina preventiva e social, atingindo um total de 82 residentes. Existe também, a residência multiprofissional, implementada em 2009, que é formada pelos graduados em: educação física, enfermagem, farmácia, fisioterapia, nutrição, psicologia, e serviço social.

Em adição, o quantitativo de colaboradores da área de enfermagem do HUGV, conforme dados do Serviço de Gestão de Pessoas em maio/2010, era de 63 cargos ocupados de enfermeiro, 194 ocupantes do cargo de técnico de enfermagem e 75 ocupantes do cargo de auxiliar de enfermagem. Desse total, estão lotados no centro cirúrgico 46 profissionais. Deve-se ressaltar que o corpo de enfermagem desempenha ainda outras funções importantes na dinâmica do hospital universitário entre elas: presença em tempo integral, familiaridade com as regras estabelecidas e os procedimentos de toda natureza, habilidade em desenvolver relacionamento informal com os outros profissionais, estreita associação com os doentes e médicos.

\section{METODOLOGIA}

Todos os respondentes assinaram o termo de consentimento livre e esclarecido, aprovado pelo Comitê de Ética em Pesquisa da Universidade Federal do Amazonas (UFAM), que informava sobre os objetivos da pesquisa e o uso dos dados, garantindo-se assim o anonimato e o caráter voluntário da participação, de igual forma, o projeto foi analisado e aprovado pela Comissão de Ensino e Pesquisa do HUGV.

Este trabalho está fundamentado em pesquisa de campo, do tipo interpretativo com método de abordagem quantitativa, cujo cenário de estudo foi o centro cirúrgico do HUGV. Foram distribuídos 46 
questionário aos servidores técnico-administrativos da equipe de enfermagem do centro cirúrgico, constituída de enfermeiros, técnicos e auxiliares de enfermagem. Destes, 37 pessoas devolveram o instrumento devidamente respondido.

Primeiramente, foi realizado um levantamento bibliográfico com base em livros e artigos, em seguida o material coletado foi utilizado como instrumento para construção de um questionário autoadministrado com 60 questões envolvendo a temática em análise. O tema foi dividido em 10 dimensões consideradas importantes para análise do clima. O instrumento de pesquisa apresenta ainda espaço para comentários e observações por parte dos participantes, pois Vergara (2005, p.13) enfatiza "os questionários estruturados, testes e escalas são os principais instrumentos de coletas de dados". Posteriormente, os dados coletados foram interpretados estatisticamente através da análise das frequências e percentuais, gerados no Software SPSS (Statistical Package for the Social Sciences) versão 16 for Windows, dessa forma, a pesquisadora seguiu as recomendações para obtenção dos resultados da pesquisa, com base na experiência vivenciada neste caso prático.

Tal sistemática consta de quatro fases básicas: construção do instrumento de medição; cálculo e construção gráfica das frequências e percentuais, verificação das correlações; e análise dos resultados obtidos.

Sendo assim, o instrumento construído foi do tipo escala atitudinal (LIKERT, 1967, p.47) oferecendo um universo de respostas que abrangeu as possíveis percepções dos servidores frente às práticas administrativas adotadas pela organização estudada.

\section{RESULTADOS}

Verificou-se que, as 60 questões distribuídas em dez dimensões, bem como, a amostra mostrou-se adequada para se alcançar os objetivos do 
estudo, uma vez que atendiam aos critérios propostos. A insatisfação dos participantes relacionados à segurança e condições no trabalho, foi um fator evidenciado, conforme tabela a seguir.

\begin{tabular}{llll}
\hline & & Frequência & \\
\hline Escala de & $\begin{array}{l}\text { Concordo Plenamente } \\
\text { (CP) }\end{array}$ & 3 & Percentual \\
Responde & $\begin{array}{l}\text { Inclinado a Concordar } \\
\text { ntes }\end{array}$ & 7 & 18,9 \\
& $\begin{array}{l}\text { IC) } \\
\text { Inclinado a Discordar }\end{array}$ & 7 & 18,9 \\
& $\begin{array}{l}\text { (ID) } \\
\text { Discordo Plenamente }\end{array}$ & 20 & 54,1 \\
\hline & (DP) & 100,0 \\
\hline
\end{tabular}

Tabela 1 - Existe a preocupação da instituição com a segurança no trabalho.

Os dados da Tabela 1 apontaram que 54,1\% dos participantes discordaram plenamente da preocupação da instituição com a segurança no trabalho.

\begin{tabular}{llll}
\hline & & Frequência & \\
\hline Escala de & $\begin{array}{l}\text { Concordo Plenamente } \\
\text { (CP) }\end{array}$ & 3 & Percentual \\
\hline $\begin{array}{l}\text { Inclinado a Concordar } \\
\text { (IC) }\end{array}$ & 4 & 10,8 \\
& $\begin{array}{l}\text { Inclinado a Discordar } \\
\text { (ID) }\end{array}$ & 17 & 45,9 \\
$\begin{array}{l}\text { Discordo Plenamente } \\
\text { (DP) }\end{array}$ & 13 & 35,1 \\
\hline
\end{tabular}

Tabela 2 - Percepção quanto à existência das condições necessárias para desenvolver o trabalho com segurança.

Os dados da Tabela 2 mostraram que 81\% dos participantes somados percentuais de inclinado a discordar e discordo plenamente observaram que a instituição não oferece as condições necessárias para os 
servidores trabalharem com segurança.Outro ponto analisado que exige uma maior atenção é a questão relacionada às condições de trabalho, pois, $50 \%$ dos servidores se sentem mal equipados durante as rotinas de trabalho desempenhadas no HUGV.

\begin{tabular}{|c|c|c|c|}
\hline & & \multirow[t]{2}{*}{ Frequência } & \multirow[b]{2}{*}{ Percentual } \\
\hline & & & \\
\hline Escala de & $\begin{array}{l}\text { Inclinado a } \\
\text { Concordar } \\
\text { (IC) }\end{array}$ & 3 & 8,3 \\
\hline \multirow[t]{4}{*}{$\begin{array}{l}\text { Respondent } \\
\text { es }\end{array}$} & $\begin{array}{l}\text { Inclinado a } \\
\text { Discordar } \\
\text { (ID) }\end{array}$ & 12 & 33,3 \\
\hline & $\begin{array}{l}\text { Discordo } \\
\text { Plenamente } \\
\text { (DP) }\end{array}$ & 21 & 58,3 \\
\hline & $\begin{array}{l}\text { Total de } \\
\text { Respondentes }\end{array}$ & 36 & 100,0 \\
\hline & Perdas & 1 & \\
\hline \multicolumn{2}{|c|}{ Total de Participantes } & 37 & \\
\hline
\end{tabular}

$\mathrm{Na}$ Tabela 3 observa-se que 58,3\% dos participantes discordaram plenamente da existência de conformidade nos itens ruído, iluminação, temperatura e poluição.

\begin{tabular}{llll} 
& & Frequência & Percentual \\
\hline Escala de & $\begin{array}{l}\text { Concordo } \\
\text { Plenamente (CP) }\end{array}$ & 3 & 8,1 \\
Respondentes & 10 & 27,0 \\
& $\begin{array}{l}\text { Inclinado a } \\
\text { Concordar (IC) } \\
\text { Inclinado a }\end{array}$ & 14 & 37,8 \\
& $\begin{array}{l}\text { Discordar (ID) } \\
\text { Discordo } \\
\text { Plenamente (DP) }\end{array}$ & 10 & 27,0 \\
\hline & Total de & 37 & 100,0 \\
\hline
\end{tabular}

Tabela 4 - Percepção quanto à existência de materiais e equipamentos adequados à execução dos serviços. 
Verifica-se na Tabela 4 que 64,8\% dos participantes, somatório de inclinado a discordar e discordo plenamente observaram a inexistência de materiais hospitalar e equipamentos adequados à execução dos serviços diários.

\begin{tabular}{llll}
\hline & & Frequência & \\
\hline Escala de & $\begin{array}{l}\text { Concordo } \\
\text { Plenamente (CP) }\end{array}$ & 20 & 54,1 \\
Respondentes & $\begin{array}{l}\text { Inclinado a } \\
\text { Concordar (IC) } \\
\text { Inclinado a Discordar } \\
\text { (ID) }\end{array}$ & 2 & 35,1 \\
$\begin{array}{l}\text { Discordo Plenamente } \\
\text { (DP) }\end{array}$ & 2 & 5,4 \\
\hline & Total de Participantes & 37 & 5,4 \\
\hline
\end{tabular}

Tabela 5 - O salário recebido na instituição satisfaz as necessidades básicas.

A Tabela 5 mostra que, 54,1\% dos participantes concordaram plenamente que o salário oferecido na instituição satisfaz as necessidades básicas, somente 10,8\% indicaram as assertivas inclinados a discordar e discordo plenamente deste item.

\section{CONSIDERAÇÕES FINAIS}

As escalas concordo plenamente e inclinado a concordar, denotam percepções positivas dos participantes em relação a certas questões que avaliaram o clima organizacional no HUGV, entre as quais se destaca: o respondente indicaria o HUGV para um amigo trabalhar; existe satisfação quanto a remuneração recebida;muitos apontaram que trabalham com prazer;entendem que são respeitados na profissão e observaram que o paciente quando bem acompanhado pela enfermagem restabelece a saúde em tempo hábil. Por outro lado, as escalas inclinadas a discordar e discordo plenamente, indicam percepções negativas, entre elas: insatisfação quanto 
as condições de trabalho e em relação a segurança no trabalho;escassez de treinamentos e de reuniões dentro do setor, bem como, falta de reconhecimento quando o trabalho é bem realizado.

Em adição, foi possível observar que os materiais hospitalares e equipamentos estão disponíveis para as rotinas de enfermagem dentro do centro cirúrgico, em níveis mínimos para atender às reais necessidades do referido centro. Além disso, foram indicadas pelos participantes a falta de materiais médico-cirúrgico adequados, bem como a escassez de equipamento de proteção individual e a existência de equipamentos ultrapassados, podendo gerar algum tipo de acidente de trabalho, e dificuldades nos momentos das cirurgias.

Por outro lado, analisando o nível de satisfação da equipe de enfermagem do centro cirúrgico, foi possível detectar que os colaboradores realizam com prazer os serviços escalados, uma vez que, demonstraram gostar do que fazem, por conseguinte, motivam-se para o trabalho, todavia, há uma insatisfação dos funcionários relacionada à escassez de treinamentos oferecidos à equipe do centro cirúrgico; percebem que não há reconhecimento por parte da chefia quando as atividades são bem realizadas; nem consideração em relação ao esforço de não parar as cirurgias quando falta material e não existe abertura para tratar com o chefe imediato uma decisão tomada.

Em suma, qualquer tentativa de melhoria do clima organizacional no HUGV, requer avanços, revisões nos processos, investimentos em equipamentos, novas posturas e recompreender o próprio papel de um hospital-escola. Os fatores em discordância evidenciados nesta pesquisa favorecem um desempenho desestruturado, desmotivação e ausência de um bom clima organizacional, gerando prejuízos a todos que direta ou indiretamente necessitam do HUGV. A importância do tema pesquisado é fundamental para a instituição, poder traçar estratégias que melhorem a 
estrutura, os equipamentos, as relações de trabalho e atinja o restabelecimento do paciente.

Espera-se que os resultados apresentados neste trabalho possam contribuir para reflexão sobre a qualidade de vida nos hospitais escolas e a importância de um bom clima organizacional no âmbito das unidades hospitalares públicas. Além disso, deve servir como um alerta ao poder público para que alguma atitude, seja tomada, em relação a escassez dos equipamentos de proteção individual, a falta de treinamentos, bem como, de medicamentos. Como temas para futuras pesquisas, sugerem-se a ampliação da amostra de análise, incluindo outros segmentos profissionais, bem como outros hospitais públicos.

\section{Referências}

BACKES, D. S.; FILHO, W. D. L.; LUNARDI, V. L. A Construção de um Ambiente de Cuidado Humano:percepção dos integrantes do grupo de humanização. Revista Nursing. Portugal, v.101, n.9, p. 1057-1063, out. 2006.

BAGGIO, M. A. Relações humanas no ambiente de trabalho: o (dês) cuidado de si do profissional de enfermagem. Revista Gaúcha de Enfermagem. v. 28, n. 3, p. 409-415, 2007.

BOOG, M.; BOOG, G. G. (Coord.). Manual de Gestão de Pessoas e Equipes. v 2. São Paulo: Gente, 2002.

BRITO, R. M. 100 anos UFAM. Manaus: EDUA, 2009.

CHIAVENATO, I. Gestão de Pessoas. $7^{\mathrm{a}}$ reimpressão. Rio de Janeiro: Elsevier, 2005.

COHEN, A. R.; FINK, S. L. Comportamento Organizacional:conceitos e estudos de caso. Maria José Cyhlar Monteiro (tradução). $7^{\mathrm{a}}$ ed. Rio de Janeiro: Campus, 2003.

DEJOURS, C.; ABDOUCHELI, E.; JAYET, C. Psicodinâmica do Trabalho. Contribuições da Escola Dejouriana a Analise da Relação Praz̧er, Sofrimento e Trabalho. (Coord.) Maria Irene StoccoBetiol. São Paulo: Atlas, 2007.

DIAS, R. Cultura Organizacional.Coleção Administração \& Sociedade. São Paulo: Alínea, 2003.

DRUCKER, P. Sociedade Pós-capitalista. $7^{\mathrm{a}}$ ed. Tradução de Nivaldo Montingelli Jr. Coleção Novos Umbrais. São Paulo: Pioneira, 1999. 
GALVÃO, M. D. A História da Medicina em Manaus. Série. Em Busca da Identidade Regional. Manaus: Valer, 2003.

GONÇALVES, E. L. Administração de Recursos Humanos nas Instituições de Saúde.São Paulo: Pioneira, 1987.

LIKERT, R. The Method of Constructing na Attitude Scale.New York, Wiley, 1967.

LONDOÑO, M.; MORERA, G.; LAVERDE, P. Administração Hospitalar. $2^{\mathrm{a}}$ ed. Rio de Janeiro: Guanabara Koogan S.A., 2003.

LOPES, N. D.Comunicação Gerencial em Enfermagem. Manaus: EDUA, 2006.

KANAANE, R. Comportamento humano nas organizações: o homem rumo ao século XXI. $2^{a}$ ed. São Paulo: Atlas, 2012.

MARQUIS, B. L.; HUSTON, C. J. Administração e Liderança em Enfermagem: teoria e prática. $4^{\mathrm{a}}$ ed. Porto Alegre: Artmed, 2005.

MARX, L. C. Manual de Gerenciamento de Enfermagem. $2^{\mathrm{a}}$ ed. Revisada e Atualizada. São Paulo: EPUB, 2003.

MOTTA, F. C. P.; VASCONCELOS, I. G. Teoria Geral da Administração. $3^{\text {a }}$ ed. rev. São Paulo: Cengage Learning, 2008.

ROBBINS, S. P. Comportamento organizacional. $11^{\mathrm{a}}$ ed. Reynaldo Marcondes (Tradução). São Paulo: Pearson Prentice Hall, 2005.

SÁ, M. A. D. de.; LEMOINE, C. Em matéria de comprometimento na empresa são os valores individuais que contam. ENANPAD. 1999.

SROUR, R. H. Poder, cultura e ética nas organizações. Rio de Janeiro: Elsevier, 1998.

STONER, J. A. F.; FREEMAN, R E. Administração. $5^{\mathrm{a}}$ ed. Alves Calado (Tradução). Rio de Janeiro: JC, 1999.

TACHIZAWA, T.; FERREIRA, V. C. P.; FORTUNA, A. A. M. Gestão com pessoas: uma abordagem aplicada às estratégias de negócios. $2^{\mathrm{a}}$ ed. Rio de Janeiro: FGV, 2001.

UNIVERSIDADE DO AMAZONAS. Regimento. Hospital Universitário Getúlio Vargas. Resolução 001/95 do Conselho de Administração da UA. 1995.

VERGARA, S. C. Projetos e Relatórios de Pesquisa em Administração. 6a ed. São Paulo: Atlas, 2005.

ZANON, U. Qualidade da Assistência Médico - Hospitalar. Conceito, Avaliação e Discussão dos Indicadores de Qualidade. Rio de Janeiro: Medsi, 2001. 